\section{Systemic Autoimmunity in a Patient With CANDLE Syndrome}

Yamazaki-Nakashimada MA ${ }^{1}$, Santos-Chávez EE ${ }^{2}$, de Jesus AA ${ }^{3}$, Rivas-Larrauri $\mathrm{F}^{1}$, Guzmán-Martínez $\mathrm{MN}^{2}$, Goldbach-Mansky $\mathrm{R}^{3}$, Espinosa-Padilla $\mathrm{S}^{2}$, Sáez-de-Ocariz $\mathrm{MdM}^{4}$, Orozco-Covarrubias $\mathrm{L}^{4}$, Blancas-Galicia $\mathrm{L}^{2}$

${ }^{1}$ Clinical Immunology Department, National Institute of Pediatrics, Mexico City, Mexico

${ }^{2}$ Immunodeficiencies Research Unit, National Institute of Pediatrics, Mexico City, Mexico

${ }^{3}$ Translational Autoinflammatory Disease Section (TADS), Laboratory of Clinical Immunology and Microbiology (LCIM), National Institute of Allergy and Infectious Diseases, National Institutes of Health, Bethesda, MD, USA

${ }^{4}$ Dermatology Department, National Institute of Pediatrics, Mexico City, Mexico

J Investig Allergol Clin Immunol 2019; Vol. 29(1): 75-76 doi: $10.18176 /$ jiaci.0338

Key words: CANDLE syndrome. Autoinflammatory. Autoimmune. Interferonopathies. Systemic lupus erythematosus.

Palabras clave: Síndrome de CANDLE. Autoinflamatorio. Autoinmune. Interferonopatias. Lupus Eritematoso Sistémico.

Chronic atypical neutrophilic dermatosis with lipodystrophy and elevated temperature (CANDLE) syndrome is a recently described genetic autoinflammatory disease caused by proteasome-immunoproteasome dysfunction. CANDLE syndrome is characterized by the early onset of recurrent fever episodes, skin lesions, violaceous periocular edema, arthralgia, limb contractures, progressive lipodystrophy, hepatosplenomegaly, elevated acute-phase reactants (APR) and liver function test (LFT) values, chronic anemia, central nervous system (CNS) calcifications, and physical growth delay. We present a patient with CANDLE syndrome that manifested with autoinflammatory and autoimmune features [1].

A 5-year-old Mexican boy presented at our hospital in 2007. He had multiple nodular, erythematous, and painful skin lesions on the face, chest, abdomen, and extremities that first appeared at birth. Hepatosplenomegaly and recurrent fever episodes had been documented since the age of 2 years. On physical examination, the child appeared wasted, with height and weight below the third percentile and delayed language development. He had a broad forehead, bilateral epicanthal folds, a broad-based nose, thick lips, large philtrum, widened metaphysis, increased abdominal girth with painful hepatosplenomegaly, axillary and inguinal lymphadenopathy, and muscular atrophy and lipoatrophy in the extremities, with nonpitting edema in the feet. The dermatological examination revealed widespread erythematous nodules and edematous annular plaques with a purpuric center and residual hyperpigmented macules (Figure, A). Skin biopsy showed

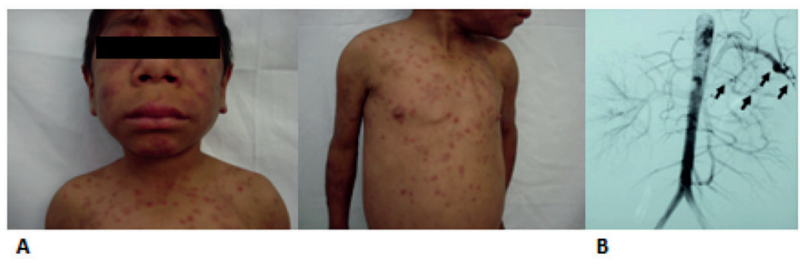

Figure. A, Widespread nodules and plaques. B, Angiogram showing renal vasculitis.

perivascular lymphohistiocytic and polymorphonuclear infiltrate with vasculitis extending into the epidermis, dermis, and subcutaneous tissue. An ophthalmological examination showed photophobia, stromal corneal edema, microaneurysms, microhemorrhages in the retina, and central diffuse leukoma in the left eye.

A complete work-up revealed chronic disease-related and autoimmune hemolytic anemia, pancytopenia, renal involvement with nephrotic syndrome, mild bilateral nephromegaly and possible cortical hamartomas, hepatic steatosis, lymphohistiocytosis, hypercholesterolemia, and persistently elevated LFT and APR values. We also observed positive antinuclear antibody (ANA) titer, hypergammaglobulinemia, negative Epstein-Barr virus (EBV) viral capsid antigen antibody IgM, positive IgG EBV nuclear antigen antibody, and $\mathrm{IgG}$ early antigen antibody. Prednisone $1 \mathrm{mg} / \mathrm{kg}$ and thalidomide were initiated, with improvement of the skin lesions.

In March 2009, the patient presented with elevated transaminases and worsening of hepatosplenomegaly and uveitis. A liver biopsy showed lymphocytic infiltrate with fibrotic areas and hepatic steatosis diagnosed as chronic hepatitis. A lymph node biopsy revealed aseptic necrotizing lymphadenopathy with a predominance of $\mathrm{CD} 8^{+} \mathrm{T}$ lymphocytes, and a skin biopsy revealed panniculitis with $\mathrm{CD}^{+}$and $\mathrm{CD} 8^{+}$ lymphocyte infiltration. Angiography revealed CNS and renal vasculitis (Figure, B). Azathioprine was added to the treatment. Seven months later, he experienced autoimmune hemolytic anemia (AIHA), immune thrombocytopenia, purpura with positive ENA-6, hypocomplementemia, and oral ulcers. Intravenous immunoglobulin, methylprednisolone pulses, and cyclosporine were administered without improvement. One month later, rituximab and mycophenolate mofetil were initiated; the blood cell count and LFT values returned to normal after 3 months.

Follow-up information was not available for 1 year. In 2013, the patient returned to the hospital with fever, anemia, leukopenia, lymphopenia, and thrombocytopenia. A physical examination revealed disseminated erythematous nodules and annular plaques, lipodystrophy affecting the cheeks and limbs, dystrophic changes in the nails, and hepatosplenomegaly. A laboratory work-up showed hypertriglyceridemia, hypergammaglobulinemia, positive titers for ANA, antineutrophil cytoplasmic antibodies (pANCA), and positive antismooth muscle antibodies. CANDLE syndrome was suspected, and mutational screening of PSMB 8 revealed the homozygous c.271G $>A$ p.A92T mutation [2]. Tocilizumab was initiated, with improvement in the LFT values and skin lesions 
(a total of 3 doses were administered). The patient was then lost to follow-up.

Autoinflammatory diseases are generally defined as sterile inflammatory conditions characterized by recurrent or persistent inflammation. In patients with periodic fever syndromes, autoantibody titers typically assessed in systemic lupus erythematosus (SLE) and rheumatoid arthritis were not present, and antigen-specific T-lymphocytes are thought not to play a pathogenic role [3].

The patient we report had evidence of adaptive immune dysregulation, which included hypergammaglobulinemia in the context of positive autoantibody titers, including elevated titers for antinuclear antibodies and lymphocytic infiltration in the liver causing autoimmune hepatitis. Some authors have noted overlapping features in patients with SLE caused by C1q deficiency and CANDLE syndrome [4]. The patient we report also presented with SLE-like features, with painless oral ulcers, proteinuria, positive antinuclear antibody titer, positive anti-ß2-GP1 titer, hypocomplementemia, autoimmune hemolytic anemia, and thrombocytopenia, which, based on laboratory and clinical findings meant that he fulfilled the classification criteria of the American College of Rheumatology. Similar autoimmune features that included AIHA, nephrotic syndrome, and positive lupus anticoagulant were also noted in 1 of the 3 patients in the paper by Torrelo et al [5], who described and named CANDLE syndrome. More recently, Tufekci et al [6] reported the case of a 2-yearold boy with CANDLE syndrome whose disease course was also complicated by AIHA, as occurred in the present case. Vasculopathies have been described in several monogenic autoinflammatory syndromes, perivascular infiltrates, and neutrophilic vasculitis. Interestingly, the patient reported herein presented with positive pANCA vasculitis involving the eyes, kidneys, and CNS; these manifestations have not yet been described in CANDLE syndrome.

Chronic type 1 interferon production has not only been associated with innate immune activation, but also with autoimmunity via several mechanisms. It promotes dendritic cell maturation, turns tolerogenic antigen-presenting cells into cells that do not suppress regulatory $\mathrm{T}$ cells, and induces plasma cell differentiation [7]. Type 1 interferon was recently shown to promote survival and the proinflammatory properties of transitional B cells from SLE patients [8]. Thus, interferonopathies as a group are associated with autoimmune processes. The concept of autoinflammatory diseases was initially proposed to distinguish the then-known autoimmune diseases from the 2 hereditary fever syndromes that lack the features of adaptive immune dysregulation. We recognize that immune regulatory defects in the innate immune system present overlapping immunological features of autoimmune diseases and vice versa. Diseases are, in fact, an immunologic continuum with variable degrees of interaction between the adaptive and innate immune systems, as originally proposed by McGonagle et al [9].

In conclusion, we report the case of a patient with CANDLE syndrome and a mutation in PSMB8. He also had autoimmune manifestations including AIHA, autoimmune hepatitis, and pANCA-positive vasculitis, thus illustrating that CANDLE syndrome can present with features that overlap between autoinflammatory and autoimmune diseases and that careful characterization of immune dysregulation is essential when selecting treatments that target immune dysregulation.

\section{Funding}

This work was supported by Fundación Mexicana para Niñas y Niños con Inmunodeficiencias A.C. and CONACYT (SALUD 2012-1-180910). MA Yamazaki-Nakashimada, M Saez-de-Ocariz, SE Espinosa-Padilla, and L Blancas-Galicia have SNI-CONACYT fellowships.

\section{Conflicts of Interest}

The authors declare they have no conflicts of interest.

\section{References}

1. Torrelo A. CANDLE Syndrome As a Paradigm of ProteasomeRelated Autoinflammation. Front Immunol. 2017;8:927.

2. McDermott $A$, Jesus AA, Liu Y, Kim P, Jacks J, Montealegre Sanchez GA, et al. A case of proteasome-associated autoinflammatory syndrome with compound heterozygous mutations. J Am Acad Dermatol. 2013;69(1):e29-32.

3. Tripathi SV, Leslie KS. Autoinflammatory diseases in dermatology: CAPS, TRAPS, HIDS, FMF, Blau, CANDLE. Dermatol Clin. 2013;31(3):387-404.

4. Al-Mayouf SM, AlSaleem A, AlMutairi N, AlSonbul A, Alzaid T, Alazami AM, et al. Monogenic interferonopathies: Phenotypic and genotypic findings of CANDLE syndrome and its overlap with C1q deficient SLE. Int J Rheum Dis. 2018;21(1):208-13.

5. Torrelo A, Patel S, Colmenero I, Gurbindo D, Lendinez F, Hernandez A, et al. Chronic atypical neutrophilic dermatosis with lipodystrophy and elevated temperature (CANDLE) syndrome. J Am Acad Dermatol. 2010;62(3):489-95.

6. Tufekci O, Bengoa S, Karapinar TH, Ataseven EB, Irken G, Oren H. CANDLE syndrome: a recently described autoinflammatory syndrome. J Pediatr Hematol Oncol. 2015;37(4):296-9.

7. Aringer $M$, Gunther $C$, Lee-Kirsch MA. Innate immune processes in lupus erythematosus. Clin Immunol. 2013;147(3):216-22.

8. Liu M, Guo Q, Wu C, Sterlin D, Goswami S, Zhang Y, et al. Type I interferons promote the survival and proinflammatory properties of transitional B cells in systemic lupus erythematosus patients. Cell Mol Immunol. 2018.

9. McGonagle D, Aziz A, Dickie LJ, McDermott MF. An integrated classification of pediatric inflammatory diseases, based on the concepts of autoinflammation and the immunological disease continuum. Pediatr Res. 2009;65(5 Pt 2):38R-45R.

Manuscript received July 25, 2018; accepted for publication October 10, 2018

Lizbeth Blancas-Galicia

9th floor, Av. Iman \#1

Insurgentes-Cuicuilco

Mexico City, Zip 04530

Mexico

E-mail: blancas.lizbeth@gmail.com 